\title{
The proper geometric dimension of the mapping class group
}

\author{
JAVIER ARAMAYONA \\ CONCHITA MARTÍNEZ-PÉREZ
}

\begin{abstract}
We show that the mapping class group of a closed surface admits a cocompact classifying space for proper actions of dimension equal to its virtual cohomological dimension.
\end{abstract}

20F34, 20F65; 20J05

\section{Introduction}

Let $\Gamma_{g, n}$ be the mapping class group of a connected orientable surface of genus $g$ with $n$ marked points. In this note we are interested in the minimal dimension $\operatorname{gd}\left(\Gamma_{g, n}\right)$ of a classifying space $\mathrm{E} \Gamma_{g, n}$ for proper actions of $\Gamma_{g, n}$. Recall that, given a discrete group $G$, the space $\underline{\mathrm{E}} G$ is a contractible space on which $G$ acts properly, and such that the fixed point set of a subgroup $H<G$ is contractible if $H$ is finite, and is empty otherwise.

Since $\Gamma_{g, n}$ is virtually torsion-free, its virtual cohomological dimension $\operatorname{vcd}\left(\Gamma_{g, n}\right)$ is a lower bound for $\operatorname{gd}\left(\Gamma_{g, n}\right)$; we remark, however, that there are groups for which the inequality is strict (Leary and Nucinkis [12]). In [7], Harer computed $\operatorname{vcd}\left(\Gamma_{g, n}\right)$ for all $g, n \geq 0$; see Theorem 2.1 below. A central ingredient of Harer's argument is the construction, for $n>0$, of a cocompact $\Gamma_{g, n}$-equivariant deformation retract (a spine) of Teichmüller space $\mathcal{T}_{g, n}$, of dimension $\operatorname{vcd}\left(\Gamma_{g, n}\right)$. Work of Penner [18] and Hensel, Osajda and Przytycki [8] shows that Harer's spine is in fact an $\underline{E} \hat{\Gamma}_{g, n}$ for the pure mapping class group $\widehat{\Gamma}_{g, n}$.

On the other hand, the case of closed surfaces of genus $g \geq 2$ is far from well-understood. Ji and Wolpert [10] used the fact that the Teichmüller space $\mathcal{T}_{g, n}$ is an $\underline{E} \Gamma_{g, n}$ to prove that the thick part $\mathcal{T}_{g, n}^{\geq \epsilon}$ of Teichmüller space is a cocompact $\underline{\mathrm{E}} \Gamma_{g, n}$ for all $g, n \geq 0$ (see also Broughton [4] and Mislin [17] for an alternative construction). Ji [9] has recently exhibited cocompact spines of $\mathcal{T}_{g, 0}$ of dimension less than $\operatorname{dim}\left(\mathcal{T}_{g, 0}\right)$ - but also greater than $\operatorname{vcd}\left(\Gamma_{g, 0}\right)-$ that serve as cocompact models of $\underline{E} \Gamma_{g, 0}$. However, it is not known whether there is a cocompact spine of $\mathcal{T}_{g, 0}$ of optimal dimension; see [3, Question 1.1]. 
More generally, Bridson and Vogtmann have asked whether if it possible to construct a cocompact $\underline{\mathrm{E}} \Gamma_{g, 0}$ of dimension equal to $\operatorname{vcd}\left(\Gamma_{g, 0}\right)$; see [3, Question 2.3]. The purpose of this note is to prove the existence of such $\underline{\mathrm{E}} \Gamma_{g, 0}$ :

Theorem 1.1 For any $g \geq 0$ there exists a cocompact $\underline{\mathrm{E}} \Gamma_{g, 0}$ of dimension equal to $\operatorname{vcd}\left(\Gamma_{g, 0}\right)$. In other words, $\operatorname{gd}\left(\Gamma_{g, 0}\right)=\operatorname{vcd}\left(\Gamma_{g, 0}\right)$.

The main tool of our proof is the algebraic invariant $\underline{\mathrm{cd}}\left(\Gamma_{g, n}\right)$ (see Section 3 ), which serves as the algebraic counterpart of $\operatorname{gd}\left(\Gamma_{g, n}\right)$. These two invariants are related in the same way as the ordinary cohomological dimension of a group $G$ is related to the minimal dimension of an EG. For example, generalizing what happens in the torsionfree case, Lück [13] proved the following Eilenberg-Ganea-type theorem, which will play a central role in our proof:

Theorem 1.2 [13] Let $G$ be a group with $\underline{\operatorname{cd}}(G)=d \geq 3$. Then there is a $d-$ dimensional $\underline{\mathrm{E}} G$. Moreover, if $G$ has a cocompact $\underline{\mathrm{E}} G$ then it also admits a cocompact $\underline{\mathrm{E}} G$ of dimension $d$.

In the light of Lück's theorem, we will prove that $\underline{\operatorname{cd}}\left(\Gamma_{g, 0}\right)=\operatorname{vcd}\left(\Gamma_{g, 0}\right)$ whenever $g \geq 3$, using a result of the second author stated as Theorem 3.3 below. The case $g \leq 2$ will require separate treatment.

Recall that the mapping class group $\Gamma_{g, n}^{b}$ of a surface with $b>0$ boundary components is torsion-free, and therefore $\underline{\operatorname{gd}}\left(\Gamma_{g, n}^{b}\right)=\underline{\operatorname{cd}}\left(\Gamma_{g, n}^{b}\right)=\operatorname{vcd}\left(\Gamma_{g, n}^{b}\right)$, by Lück's Theorem 1.2. This fact, together with Theorem 1.1, plus a minor extension of our arguments (see Remark 4.5 below) yields the following:

Corollary 1.3 For all $g, n, b \geq 0$, there exists a cocompact $\underline{\mathrm{E}} \Gamma_{g, n}^{b}$ of dimension equal to $\operatorname{ved}\left(\Gamma_{g, n}^{b}\right)$.

\section{Acknowledgements}

The authors would like to thank Chris Leininger, Greg McShane and Juan Souto for their conversations.

This research was supported by Gobierno de Aragón, European Regional Development Funds and MTM2010-19938-C03-03. 


\section{Preliminaries}

Let $S_{g, n}$ be a connected, orientable surface of genus $g \geq 0$, with empty boundary and $n \geq 0$ marked points. The mapping class group $\Gamma_{g, n}$ is the group of isotopy classes of orientation-preserving homeomorphisms of $S_{g, n}$, where homeomorphisms and isotopies are required to map the set of marked points to itself. For simplicity, we will write $S_{g}:=S_{g, 0}$ and $\Gamma_{g}:=\Gamma_{g, 0}$.

\subsection{Virtual cohomological dimension}

Recall that $\Gamma_{g, n}$ has a torsion-free subgroup of finite index. As mentioned earlier, Harer [7] computed the virtual cohomological dimension $\operatorname{vcd}\left(\Gamma_{g, n}\right)$ of $\Gamma_{g, n}$ :

Theorem 2.1 (Harer) If $2 g+n>2$, then

$$
\operatorname{vcd}\left(\Gamma_{g, n}\right)= \begin{cases}4 g+n-4 & \text { if } g, n>0 \\ 4 g-5 & \text { if } n=0 \\ n-3 & \text { if } g=0\end{cases}
$$

Remark 2.2 If $n \leq 1$ then $\Gamma_{0, n}$ is trivial. Also, $\Gamma_{0,2} \cong \mathbb{Z}$ and $\Gamma_{1,0} \cong \mathrm{SL}_{2}$ ( $\mathbb{Z}$ ) (Farb and Margalit [6]). Therefore, $\operatorname{vcd}\left(\Gamma_{0, n}\right)=0$ for $n \leq 1$, and $\operatorname{vcd}\left(\Gamma_{0,2}\right)=\operatorname{vcd}\left(\Gamma_{1,0}\right)=1$.

\subsection{Riemann-Hurwitz formula}

Let $g \geq 2$. By the Nielsen realization theorem [11], every finite subgroup of $\Gamma_{g}$ may be realized as a group of isometries with respect to some hyperbolic metric on $S_{g}$. Therefore, given a finite subgroup $L \leq \Gamma_{g}$, and slightly abusing notation, we may consider the (hyperbolic) orbifold $S_{g} / L$. We denote by $g_{L}$ the genus of $S_{g} / L$; similarly, let $k_{L}$ be the number of orbifold points of $S_{g} / L$, of orders $p_{1}^{L}, \ldots, p_{k_{L}}^{L}$, respectively. The tuple $\left(g_{L} ; p_{1}^{L}, \ldots, p_{k_{L}}^{L}\right)$ is called the signature of $L$. Since $S_{g} \rightarrow S_{g} / L$ is an orbifold cover of degree $|L|$, the multiplicativity of the orbifold Euler characteristic implies that $g$ and the signature of $S_{g} / L$ are related by the so-called Riemann-Hurwitz formula; see eg [6]:

$$
\frac{2 g-2}{|L|}=2 g_{L}-2+l_{L}
$$

where

$$
l_{L}=\sum_{i=1}^{k_{L}}\left(1-\frac{1}{p_{i}^{L}}\right)
$$


Observe that (2) implies

$$
\frac{k_{L}}{2} \leq l_{L} \leq k_{L}
$$

We will need the following surely well-known observation:

Lemma 2.3 Let $L<T$ be two distinct finite subgroups of $\Gamma_{g}$, with $g \geq 2$, and denote by $\left(g_{L} ; q_{1}, \ldots, q_{k_{L}}\right)$ and $\left(g_{T} ; p_{1}, \ldots, p_{k_{T}}\right)$ the signatures of $S / L$ and $S / T$, respectively. Then:

(i) If $g_{T}>1$ then $g_{T}<g_{L}$.

(ii) If $g_{T} \leq 1$ then $g_{T} \leq g_{L}$; moreover, if $g_{T}=g_{L}$ then $k_{T}<k_{L}$.

Proof The map $S_{g} / L \rightarrow S_{g} / T$ is an orbifold cover of degree $d=[T: L]>1$. By the Riemann-Hurwitz formula, we have

$$
2-2 g_{L}+\sum_{i=1}^{k_{L}} 1 / q_{i}-k_{L}=d\left(2-2 g_{T}+\sum_{i=1}^{k_{T}} 1 / p_{i}-k_{T}\right) .
$$

Now

$$
\sum_{i=1}^{k_{L}} 1 / q_{i}=d \sum_{i=1}^{k_{T}} 1 / p_{i}
$$

(see, for instance, [6, Section 7.2.2]). Hence

$$
2 g_{L}+k_{L}-2=d\left(2 g_{T}-2\right)+d k_{T} .
$$

We prove the first claim of the lemma. Assume that $g_{T}>1$ and suppose, for contradiction, that $g_{L} \leq g_{T}$. From (4),

$$
d\left(2 g_{T}-2\right)+d k_{T}=2 g_{L}-2+k_{L} \leq 2 g_{T}-2+d k_{T},
$$

which implies that $d\left(2 g_{T}-2\right) \leq 2 g_{T}-2$, a contradiction since $d>1$. We have thus proved part (i).

Moving on to the second claim of the lemma, assume $g_{T} \leq 1$. We first prove that $g_{T} \leq g_{L}$. Arguing again by contradiction, the only case to rule out is $g_{T}=1$ and $g_{L}=0$. From (4), we get: $k_{L}-2=d k_{T} \geq k_{L}$, which is impossible. Hence $g_{L} \leq g_{T}$, as claimed.

Finally, we prove that $k_{T}<k_{L}$ whenever $g_{T}=g_{L}$; recall that the latter implies $g_{T} \in\{0,1\}$. First, if $g_{T}=1$, (4) gives $k_{L}=d k_{T}$, and thus $k_{T}<k_{L}$, as desired. If $g_{T}=0$, again (4) yields $k_{L}-2=d\left(k_{T}-2\right)$, which gives $k_{T}<k_{L}$ as well (observe that $k_{L}, k_{T}>2$ since $\left.g_{T}=g_{L}=0\right)$. This finishes the proof of the lemma. 


\section{Preliminaries on classifying spaces for proper actions}

As mentioned in the introduction, we will determine $\underline{\operatorname{gd}}\left(\Gamma_{g, n}\right)$ using the algebraic invariant $\underline{\mathrm{cd}}\left(\Gamma_{g, n}\right)$, which is defined along the same lines as the ordinary cohomological dimension but in the setting of proper actions. Informally, it is the length of the shortest projective resolution of the trivial object in a certain category, whose objects are called Bredon modules. Here, we will only need to make use of two facts about $\underline{\operatorname{cd}}(G)$, referring the reader to Lück $[13 ; 14]$ for a discussion on $\underline{\operatorname{cd}}(G)$.

The first fact about $\underline{\mathrm{cd}}(G)$ that we will need is Lück's Theorem 1.2, which is a consequence of [13, Theorem 13.19]. A proof of the existence of the model in Theorem 1.2 was given by Brady, Leary and Nucinkis [2]; we now explain how to adapt their argument to produce a cocompact one.

Let $X$ be a cocompact $\underline{\mathrm{E}} G$ of dimension $d$. The $(d-1)$-skeleton $Z$ of $X$ gives a chain complex of free Bredon modules, which is exact except possibly in degree $d-1$. Let $M$ be the $(d-1)^{\text {th }}$ homology group of $Z$. As in the classical case - see Brown [5, Section VIII, Lemma 2.1] $-M$ is a projective Bredon module, which is finitely generated since $X$ is cocompact. Note that $M$ might not be free. However, the versions for Bredon modules, see [5, Section VIII, Lemma 4.4 and Proposition 6.5], together imply that there is some free Bredon module $P$ such that $F:=P \oplus M$ is free and that $P$ can be taken to be finitely generated, so that $F$ is also finitely generated. A finitely generated free Bredon module is determined by a finite family of representatives of conjugacy classes of finite subgroups. Denote by $\Lambda_{P}, \Lambda_{F}$, respectively, the families for $P$ and $F$. Now, attach to $Z$ orbits of $(d-1)$-cells of types $S^{d-1} \times G / H$ for $H \in \Lambda_{P}$, and use Hurewicz's Theorem to attach orbits of $d$-cells of types $S^{d} \times G / H$ for $H \in \Lambda_{F}$. This way we get a new cocompact CW-complex $Y$ such that the fixed point set of $L<G$ is contractible whenever $L$ is finite and empty otherwise. In other words, $Y$ is the desired model for $\underline{E} G$.

Remark 3.1 Theorem 1.2 also holds if $d=1$; see [2].

Before we describe the second property of $\underline{\operatorname{cd}}(G)$ that will be used, we need some definitions. Consider, for every finite subgroup $H<G$, the Weyl group

$$
W H:=N_{G}(H) / H,
$$

where $N_{G}(H)$ denotes the normalizer of $H$ in $G$. Observe that the centralizer $Z_{G}(H)$ of $H$ has finite index in $N_{G}(H)$, and thus $W H$ and $Z_{G}(H)$ are weakly commensurable. Let $\mathcal{F}_{H}=\{T \leq G$ finite $\mid H<T\}$, noting that the group $W H$ acts on the poset $\mathcal{F}_{H}$ by conjugation. 
Let $\mathcal{F}_{H} \bullet$ be the chain complex of $G$-modules associated to the geometric realization of $\mathcal{F}_{H}$, and let $\Sigma \widetilde{\mathcal{F}}_{H} \bullet$ be the result of augmenting and suspending $\mathcal{F}_{H} \bullet$ Finally, write $\operatorname{pd}_{W H} \Sigma \widetilde{\mathcal{F}}_{H \bullet}$ for the projective dimension of the chain complex $\Sigma \widetilde{\mathcal{F}}_{H \bullet}$, namely the shortest length of a chain complex $P_{\bullet}$ of projective $G$-modules such that there is a morphism $P_{\bullet} \rightarrow \Sigma \widetilde{\mathcal{F}}_{H \bullet}$ inducing an isomorphism in the homology groups.

A result of Connolly and Kozniewski, stated as [16, Theorem A], implies

$$
\underline{\operatorname{cd}}(G)=\max _{H \leq G \text { finite }} \operatorname{pd}_{W H} \Sigma \tilde{\mathcal{F}}_{H \bullet} .
$$

We will need:

Definition 3.2 (Length) The length $\lambda(L)$ of a finite group $L$ is the largest number $i \in \mathbb{N} \cup\{0\}$ for which there is a sequence $1=L_{0}<L_{1}<\cdots<L_{i}=L$.

We are finally ready to introduce the promised second fact about $\underline{\mathrm{cd}}$, which follows as an easy consequence of (5):

Theorem 3.3 Let $G$ be a virtually torsion-free group such that for any $H \leq G$ finite, $\operatorname{vcd}(W H)+\lambda(H) \leq \operatorname{vcd}(G)$. Then $\underline{\operatorname{cd}}(G)=\operatorname{vcd}(G)$.

Proof For $i \geq 0$, the $i^{\text {th }}$ term of $\Sigma \widetilde{\mathcal{F}}_{H \bullet}$ is the permutation module associated to the action of $G$ on the cells of the form $T:=H_{i}>\cdots>H_{1}>H_{0}=H$, whose stabilizer is weakly commensurable with $W T$. Observe that $i+\lambda(H) \leq \lambda(T)$. Therefore

$$
\operatorname{pd}_{W H} \Sigma \widetilde{\mathcal{F}}_{H \bullet} \leq \max \left\{\lambda(T)-\lambda(H)+\operatorname{vcd} W T \mid T \in \mathcal{F}_{H} \cup\{H\}\right\} \leq \operatorname{vcd}(G) .
$$

Using (5) we get $\underline{\operatorname{cd}}(G) \leq \operatorname{vcd}(G)$; the other inequality is well known.

\section{Proof of Theorem 1.1}

In the light of Theorem 3.3, we are going to need to understand the relation between $\operatorname{vcd}\left(\Gamma_{g}\right)$ and $\operatorname{vcd}(W L)$, for every finite subgroup $L<\Gamma_{g}$. The following is well-known; see, for instance, Maher [15, Proposition 2.3]:

Lemma 4.1 Let $L \leq \Gamma_{g}$ be a finite subgroup of signature $\left(g_{L} ; p_{1}^{L}, \ldots, p_{k_{L}}^{L}\right)$. Then $W L$ has finite index in $\Gamma_{g_{L}, k_{L}}$. In particular, $\operatorname{vcd}(W L)=\operatorname{vcd}\left(\Gamma_{g_{L}, k_{L}}\right)$.

For notation purposes, it will be convenient to write

$$
v(L):=4 g_{L}+k_{L}-4 .
$$


Observe that, from Theorem 2.1 and Lemma 4.1, we have

$$
\operatorname{vcd}(W L)= \begin{cases}v(L) & \text { if } g_{L}, k_{L}>0 \\ v(L)-1 & \text { if } k_{L}=0 \\ v(L)+1 & \text { if } g_{L}=0\end{cases}
$$

We will need:

Proposition 4.2 Let $L<T$ be finite subgroups of $\Gamma_{g}$, where $g \geq 2$. Assume that $g_{T}<g_{L}$. Then $\operatorname{vcd}(W T)<\operatorname{vcd}(W L)$, unless we are in one of the following two cases:

(i) $\left(g_{L}, k_{L}\right)=(2,0)$ and $\left(g_{T}, k_{T}\right)=(0,6)$.

(ii) $\left(g_{L}, k_{L}\right)=(1, r)$ and $\left(g_{T}, k_{T}\right)=(0, r+3)$, for some $r \geq 1$.

Proof First, observe that $L<T$ implies $Z_{\Gamma_{g}}(T) \leq Z_{\Gamma_{g}}(L)$, and thus $\operatorname{vcd}(W T) \leq$ $\operatorname{vcd}(W L)$. Using (3) and the Riemann-Hurwitz formula we deduce that

$$
\begin{aligned}
v(T)=4 g_{T}-4+k_{T} & \leq 4 g_{T}-4+2 l_{T}=\frac{4 g_{L}-4+2 l_{L}}{|T: L|} \\
& \leq \frac{4 g_{L}-4+2 k_{L}}{|T: L|}=\frac{v(L)+k_{L}}{|T: L|} \leq \frac{v(L)+k_{L}}{2} .
\end{aligned}
$$

Armed with inequality (7), and noting that $g_{L}>0$, we distinguish the following cases:

Case $1\left(g_{T}>0\right)$ We have the following subcases:

(1a) $k_{T}=k_{L}=0$ : Since $g_{T}<g_{L}$ then $\operatorname{vcd}(W T)<\operatorname{vcd}(W L)$.

(1b) $k_{L}=0, k_{T} \neq 0$ : Since $g_{L} \geq 2$, we have that $v(L) \geq 4$. From (6),

$$
\operatorname{vcd}(W T)=v(T) \leq \frac{v(L)}{2}<v(L)-1=\operatorname{vcd}(W L) .
$$

(1c) $k_{L} \neq 0, k_{T}=0$ : Note that $g_{T} \geq 2$, and so $g_{L} \geq 3$. In particular, $\operatorname{vcd}(W L)=$ $4 g_{L}-4+k_{L} \geq k_{L}+8$. Therefore, using (6) and (7),

$$
\operatorname{vcd}(W T)=v(T)-1 \leq \frac{v(L)+k_{L}-2}{2}<\operatorname{vcd}(W L) .
$$

(1d) $k_{L} \neq 0, k_{T} \neq 0$ : In this case, since $0<g_{T}<g_{L}$, then $\operatorname{vcd}(W T)<\operatorname{vcd}(W L)$.

Case $2\left(g_{T}=0\right)$ Note that $k_{T}>0$. We have the following subcases:

(2a) $k_{L}=0$ : Again by (1), $g_{L} \geq 2$, and in particular $\operatorname{vcd}(W L) \geq 3$. From this, and using (6) and (7), we deduce

$$
\operatorname{vcd}(W T)-1 \leq \frac{\operatorname{vcd}(W L)+1}{2}<\operatorname{vcd}(W L)-1
$$


unless $\operatorname{vcd}(W L)=3$. In the latter case, either $\operatorname{vcd}(W T)<\operatorname{vcd}(W L)$ or $\left(g_{L}, k_{L}\right)=(2,0)$ and $\left(g_{T}, k_{T}\right)=(0,6)$, as claimed.

(2b) $k_{L} \neq 0$ : Suppose first that $g_{L} \geq 2$, in which case $\operatorname{vcd}(W L)=4 g_{L}-4+k_{L} \geq$ $k_{L}+4$. From (6) and (7), we obtain

$$
\operatorname{vcd}(W T)-1 \leq \frac{\operatorname{vcd}(W L)+k_{L}}{2} \leq \frac{2 \operatorname{vcd}(W L)-4}{2}<\operatorname{vcd}(W L)-1,
$$

and thus the result follows. Suppose now that $g_{L}<2$, and thus $g_{L}=1$ as $0=g_{T}<g_{L}$. As vcd $W T \leq \operatorname{vcd} W L$, in the equality case we have

$$
k_{L}=\operatorname{vcd}(W L)=\operatorname{vcd}(W T)=k_{T}-3,
$$

and we are in part (ii) of the theorem.

Remark 4.3 Cases (i) and (ii) in Proposition 4.2 do occur in practice. Indeed, there is a branched double-cover $S_{2,0} \rightarrow S_{0,6}^{*}$, where $S_{0,6}^{*}$ denotes a sphere with six cone points of angle $\pi$, induced by the hyperelliptic involution of $S_{2,0}$. By a result of Birman and Hilden [1], we may realize $\Gamma_{0,6}$ as a subgroup of index 2 in $\Gamma_{2,0}$. Along similar lines, $\Gamma_{0,5}$ is a subgroup of index 2 in $\Gamma_{1,2}$, arising from the hyperelliptic involution of $S_{1,2}$.

The next result is the key technical observation of this note:

Proposition 4.4 If $g \geq 3$, then for any $T<\Gamma_{g}$ finite,

$$
\operatorname{vcd}(W T)+\lambda(T) \leq \operatorname{vcd}\left(\Gamma_{g}\right) .
$$

Proof Our first objective is to establish the following:

Claim Let $1 \neq T<\Gamma_{g}$ be finite, where $g \geq 3$. If $g_{T}>0$, then

$$
\operatorname{vcd}(W T)+\lambda(T)+1 \leq \operatorname{vcd}\left(\Gamma_{g}\right) .
$$

Proof of Claim Using (1) and (3), and since $g_{T}>0$, we have

$$
\begin{aligned}
\frac{\operatorname{vcd}\left(\Gamma_{g}\right)+1}{|T|} & =\frac{4 g-4}{|T|}=4 g_{T}-4+2 l_{T} \geq 4 g_{T}-4+k_{T} \\
& =v(T) \geq \operatorname{vcd}(W T) .
\end{aligned}
$$

Rearranging, we obtain $\operatorname{vcd}\left(\Gamma_{g}\right) \geq|T| \operatorname{vcd}(W T)-1$. In particular, observe that (8) is satisfied whenever

$$
\operatorname{vcd}(W T)+\lambda(T)+2 \leq|T| \operatorname{vcd}(W T)
$$


holds. We distinguish the following cases, depending on the value of $\operatorname{vcd}(W T)$ :

(i) If $\operatorname{vcd}(W T) \geq 3$, then 4 is true for all finite subgroups $T \leq \Gamma_{g}$, as $\lambda(G) \leq|G|-1$ for every finite group $G$.

(ii) If $\operatorname{vcd}(W T)=2$, then 4 holds unless $|T|=2$, again since $\lambda(T) \leq|T|-1$. But if $|T|=2$ then $\lambda(T)=1$, and thus (8) follows because $\operatorname{vcd}\left(\Gamma_{g}\right) \geq 7$ as $g \geq 3$.

(iii) If $\operatorname{vcd}(W T)=1$ then 4 is satisfied unless $|T| \in\{2,3,4\}$. To see this, observe that if $T$ has a maximal subgroup satisfying 4 , then the same holds for $T$, and that groups of orders $8,9,6, p$ for $p$ a prime $p>3$ satisfy 4 . In the remaining cases $\lambda(T) \leq 2$, and hence (8) follows as in the previous case since $g \geq 3$.

This finishes the proof of the claim.

Returning to the proof of the proposition, let $T \leq \Gamma_{g}$ be a finite subgroup. If $T=1$ then the result is trivial, and if $g_{T}>0$, then it follows from the claim above. Therefore, assume that $T \neq 1$ and $g_{T}=0$. Let $L \leq T$ be such that $\lambda(T)=\lambda(L)+1$. Suppose first that $L=1$, noting that $\lambda(T)=1$. Since $g_{L}=g \geq 3$, Proposition 4.2 implies that $\operatorname{vcd}(W T)<\operatorname{vcd}(W L)=\operatorname{vcd}\left(\Gamma_{g}\right)$, and so we are done. Thus assume that $L \neq 1$. If $g_{L}>0$, the claim above yields

$$
\operatorname{vcd}(W T)+\lambda(T) \leq \operatorname{vcd}(W L)+\lambda(L)+1 \leq \operatorname{vcd}\left(\Gamma_{g}\right) .
$$

On the other hand, if $g_{L}=0$ then $k_{T}<k_{L}$, by Lemma 2.3. Thus

$$
\begin{aligned}
\operatorname{vcd}(W T)+\lambda(T) & =k_{T}-3+\lambda(T) \\
& <\operatorname{vcd}(W L)+\lambda(L)+1 .
\end{aligned}
$$

Hence $\operatorname{vcd}(W T)+\lambda(T) \leq \operatorname{vcd}(W L)+\lambda(L)$, and the result follows by induction on the length of $T$.

We are finally ready to prove Theorem 1.1:

Proof of Theorem 1.1 First, if $g \geq 3$, the result follows combining Lück's Theorem 1.2 with Theorem 3.3 and Proposition 4.4. If $g=0$ then $\Gamma_{0,0}=1$ so the result is trivial. Next, if $g=1$ then $\Gamma_{1,0}=\mathrm{SL}_{2}(\mathbb{Z})$ and one can take the dual tree to the Farey graph as a model of $\underline{E} \Gamma_{1,0}$. Finally, $\Gamma_{2,0}$ is a central extension of $\Gamma_{0,6}$ by $\mathbb{Z}_{2}$. Let $X$ be a cocompact model for $\underline{E} \Gamma_{0,6}$ of dimension $\operatorname{vcd}\left(\Gamma_{0,6}\right)$ - Harer's spine, for instance. Then the action of $\Gamma_{0,6}$ on $X$ can be lifted to an action of $\Gamma_{2,0}$, and thus $X$ is a cocompact model for $\underline{\mathrm{E}} \Gamma_{2,0}$ too. Since $\operatorname{vcd}\left(\Gamma_{2,0}\right)=\operatorname{vcd}\left(\Gamma_{0,6}\right)$, we are done.

Remark 4.5 An inductive argument along the lines of [17, Section 4], using the Birman short exact sequence, [14, Theorem 5.16] and Harer's formula yields the analogue of Theorem 1.1 for surfaces with a non-empty set of marked points. 


\section{References}

[1] J S Birman, H M Hilden, On isotopies of homeomorphisms of Riemann surfaces, Ann. of Math. 97 (1973) 424-439 MR0325959

[2] N Brady, IJ Leary, B E A Nucinkis, On algebraic and geometric dimensions for groups with torsion, J. London Math. Soc. 64 (2001) 489-500 MR1853466

[3] M R Bridson, K Vogtmann, Automorphism groups of free groups, surface groups and free abelian groups, from: "Problems on mapping class groups and related topics", (B Farb, editor), Proc. Sympos. Pure Math. 74, Amer. Math. Soc. (2006) 301-316 MR2264548

[4] S A Broughton, Normalizers and centralizers of elementary abelian subgroups of the mapping class group, from: "Topology '90”, (B Apanasov, W D Neumann, A W Reid, L Siebenmann, editors), Ohio State Univ. Math. Res. Inst. Publ. 1, de Gruyter, Berlin (1992) 77-89 MR1184404

[5] KS Brown, Cohomology of groups, Graduate Texts in Mathematics 87, Springer, New York (1982) MR672956

[6] B Farb, D Margalit, A primer on mapping class groups, Princeton Mathematical Series 49, Princeton Univ. Press (2012) MR2850125

[7] J L Harer, The virtual cohomological dimension of the mapping class group of an orientable surface, Invent. Math. 84 (1986) 157-176 MR830043

[8] S Hensel, D Osajda, P Przytycki, Realisation and dismantlability arXiv 1205.0513

[9] L Ji, Well-rounded equivariant deformation retracts of Teichmüller spaces arXiv: 1302.0877

[10] L Ji, S A Wolpert, A cofinite universal space for proper actions for mapping class groups, from: "In the tradition of Ahlfors-Bers, V", (M Bonk, J Gilman, H Masur, Y Minsky, M Wolf, editors), Contemp. Math. 510, Amer. Math. Soc. (2010) 151-163 MR2581835

[11] S P Kerckhoff, The Nielsen realization problem, Ann. of Math. 117 (1983) 235-265 MR690845

[12] I J Leary, B E A Nucinkis, Some groups of type VF, Invent. Math. 151 (2003) 135165 MR1943744

[13] W Lück, Transformation groups and algebraic $K$-theory, Lecture Notes in Mathematics 1408, Springer, Berlin (1989) MR1027600

[14] W Lück, Survey on classifying spaces for families of subgroups, from: "Infinite groups: geometric, combinatorial and dynamical aspects", (L Bartholdi, T CeccheriniSilberstein, T Smirnova-Nagnibeda, A Zuk, editors), Progr. Math. 248, Birkhäuser, Basel (2005) 269-322 MR2195456 
[15] J Maher, Random walks on the mapping class group, Duke Math. J. 156 (2011) 429-468 MR2772067

[16] C Martínez-Pérez, Subgroup posets, Bredon cohomology and equivariant Euler characteristics, Trans. Amer. Math. Soc. 365 (2013) 4351-4370 MR3055698

[17] G Mislin, Classifying spaces for proper actions of mapping class groups, Münster J. Math. 3 (2010) 263-272 MR2775365

[18] R C Penner, The decorated Teichmüller space of punctured surfaces, Comm. Math. Phys. 113 (1987) 299-339 MR919235

School of Mathematics, Statistics and Applied Mathematics

National University of Ireland Galway, University Road, Galway, Ireland

Departamento de Matemáticas, Universidad de Zaragoza

50009 Zaragoza, Spain

Javier.Aramayona@nuigalway.ie, conmar@unizar.es

Received: 20 February 2013 